\title{
ÉTICA E GERÊNCIA NO CUIDADO DE ENFERMAGEM
}

\author{
Cíntia da Silva Mazur ${ }^{1}$,Liliana Labronici² ${ }^{2}$ Lillian Daisy Gonçalves Wolff ${ }^{3}$
}

\begin{abstract}
RESUMO: Reflexão teórica a respeito da postura ética profissional desejável que enfermeiros devem adotar ao exercerem o papel gerencial no cuidado de enfermagem, visto que suas ações gerenciais exigem tomadas de decisão éticas em prol do bem estar do paciente. $\mathrm{O}$ atendimento às necessidades do paciente, da equipe e da instituição exige que enfermeiros tomem decisões fundamentadas na ética, porquanto esta expressa tanto os valores sociais como os pessoais construídos, elaborados e introjetados ao longo da trajetória existencial, na dimensão pessoal, social e profissional, e nela se encontram os princípios da ação. Neste sentido, a postura ética demonstrada pelo enfermeiro é de suma relevância, considerando-se que a evolução e o reconhecimento social da profissão acontece quando os profissionais comprometem-se mediante a adoção de condutas que visem melhorar a qualidade do cuidado à saúde, respeitando os princípios éticos universais referentes à preservação da dignidade humana, beneficência, não maleficência e justiça.
\end{abstract}

PALAVRAS-CHAVE: Cuidados de enfermagem; Organização \& administração; Ética.

\section{ETHICS AND MANAGEMENT IN NURSING CARE}

\begin{abstract}
Theoretical reflection on the desirable professional ethical behavior that nurses must adopt in their managerial role in nursing care once their managerial actions demand ethical decision-making on patients' welfare. Meeting patients', nursing teams', and organizations' needs urges ethical decision-making on the part of nurses, because ethics expresses both social and personal values, built, elaborated and introjected throughout the existential trajectory, in personal, social and professional dimensions; in it, the action principles are also found. Thus, an ethical behavior demonstrated by nurses is highly relevant, taking into account that nursing evolution and social recognition are verified when its professionals, by means of the adoption of certain behaviors, are committed to increasing the quality of health care by complying with universal ethical principles concerning the preservation of human dignity, welfare, harm prevention, and justice.
\end{abstract}

KEYWORDS: Nursing care; Organization \& management; Ethics.

\section{ÉTICAY ADMINISTRACIÓN EN EL CUIDADO DE LA ENFERMERÍA}

RESUMEN: Reflexión teórica acerca de la postura ética profesional que los enfermeros deben adoptar al ejercer el papel de administración en el ámbito de la enfermería. Las acciones de gerencia de los enfermeros exigen que se tomen decisiones éticas a favor del bienestar del paciente. El atendimiento a las necesidades del paciente, del equipo de trabajo y de la institución es solicitado y exige que los enfermeros tomen decisiones fundamentadas en la ética y en la moral. En este sentido, la postura ética adoptada por el enfermero es de suma relevancia, considerándose que el desarrollo y el reconocimiento social de la profesión ocurren cuando los profesionales se comprometen mediante la adopción de conductas que buscan mejorar la calidad del cuidado de la salud, respetando los principios éticos universales referentes a la preservación de la dignidad humana, a la beneficencia, a la no maleficencia y a la justicia.

PALABRAS CLAVE: Cuidados de enfermería; Organización y administración; Ética.

'Enfermeira. Especialista em Psicologia do Trabalho pela UFPR. Mestranda do Programa de Pós-graduação em Enfermagem da Universidade Federal do Paraná. Membro do NEPECHE/UFPR.

${ }^{2}$ Enfermeira. Doutora em Enfermagem pela UFSC. Membro do Grupo de Estudos Multiprofissional em Saúde do Adulto (GEMSA/UFPR). Professora do Programa de Pós-graduação em Enfermagem da Universidade Federal do Paraná.

${ }^{3}$ Enfermeira. Doutora em Engenharia da Produção pela Universidade Federal de Santa Catarina (UFSC). Professora do Curso de Graduação em Enfermagem da Universidade Federal do Paraná (UFPR. Membro do Núcleo de Estudos, Pesquisa e Extensão em Cuidado Humano (NEPECHE/ UFPR).

Autor correspondente:

Lillian Daisy Gonçalves Wolff

R.Padre Camargo, 120 - 80060-240 - Curitiba-Pr

Recebido: $29 / 10 / 2007$

Email:ldgw@ufpr.br 


\section{INTRODUÇÃO}

O processo de trabalho dos profissionais de saúde tem como finalidade a ação terapêutica de saúde; como objeto o indivíduo ou grupos doentes, sadios ou expostos a risco, que necessitam de medidas que possibilitem a preservação da saúde, a prevenção ou a cura de doenças; e, como instrumental de trabalho, os instrumentos e as condutas que representam o nível técnico do conhecimento ${ }^{(1)}$.

As relações de produção capitalista e a própria racionalidade científica moderna desenvolveram-se de maneira bastante acelerada no último século, por meio do aperfeiçoamento de técnicas para controlar e organizar a natureza ${ }^{(2)}$. Deste modo, com a modernidade, um conjunto de novas demandas e necessidades sociais foi estabelecido e passou a exigir novos mecanismos de produção em saúde, cada vez mais especializados e individualizados.

Ao considerarmos que os profissionais de saúde exercem suas atividades para atender às necessidades de indivíduos, faz-se mister levar em consideração o fato de que essas necessidades se modificam e se ampliam ao longo do tempo, motivo pelo qual é indispensável a reavaliação permanente de suas condutas, assim como atualização contínua acerca de conhecimentos técnico-científicos, e de questões políticas e sociais emergentes que influenciam as práticas em saúde.

Inserida nesse contexto de transformações constantes na sociedade globalizada, a Enfermagem como uma profissão da área da saúde não fica inerte a esse processo e evolui continuamente, em consonância com as mudanças sociais. Trata-se de "uma ciência humana, de pessoas e de experiências com campo de conhecimento, fundamentações e práticas do cuidar dos seres humanos que abrangem do estado de saúde aos estados de doença, mediada por transações pessoais, profissionais, científicas, estéticas, éticas e políticas"(3). Neste sentido, seus profissionais atuam não apenas na proteção e promoção da saúde, como na reabilitação de pessoas que necessitam de cuidados de Enfermagem.

Atualmente, o trabalho da Enfermagem no Brasil é tecnicamente dividido em três categorias: enfermeiro, técnico e auxiliar de enfermagem, e cada uma exerce suas atividades conforme o estabelecido na Lei $\mathrm{n}^{\circ} 7.498$ que regulamenta o exercício de enfermagem ${ }^{(4)}$. No seu Art. 11 do Capítulo I estabelece que "compete privativamente ao enfermeiro o planejamento, a organização, a coordenação, execução e avaliação dos serviços de assistência de enfermagem"(4).

Há que se ressaltar que o enfermeiro, no cotidiano de sua prática em instituições de saúde, é responsável pelo gerenciamento do cuidado. Este tem por finalidade proporcionar todas as condições necessárias à sua execução. Nesse contexto, seu agir é permeado pelo entrelaçamento do conhecimento técnico-científico com os conhecimentos e princípios éticos adquiridos e construídos na sua trajetória existencial e armazenados em sua bagagem cultural. Desta maneira, podemos dizer que entram em jogo valores pessoais e da profissão que exigem do enfermeiro uma reflexão e avaliação constante no desenvolvimento da sua prática profissional, tanto assistencial como gerencial.

\section{DESENVOLVIMENTO}

O enfermeiro ao exercer funções assistenciais e gerenciais fundamenta seu fazer de acordo com sua formação e competência (habilidades, atitudes e conhecimento), e nele está implícito sua formação moral e ética que dá suporte no momento da tomada de decisão, uma vez que sua prática se dá em múltiplos cenários onde estão presentes Eros e Thánatos ${ }^{(5)}$, ou seja, vida e morte, além de uma multiplicidade de atores que necessitam de cuidado, e é permeada por precariedades inúmeras que podem provocar dilemas e situações de conflito que geram desgaste.

É interessante destacar que "a ética está originariamente ligada à noção da possibilidade de se definir o bem, quer como algo que é uma realidade perfeita, quer como algo de uma realidade perfeita, quer como objeto de desejo ou de aspiração"(6:33). Com relação a essa possibilidade, a ética é compreendida como a ciência do fim ao qual a conduta humana deve dirigir-se, ao ideal a que o homem deve, por sua natureza, voltar-se; é um tipo de saber normativo que pretende orientar as ações dos seres humanos. Isto significa que ela nos leva à reflexão sobre os múltiplos modos de justificar racionalmente a vida moral. Assim, seu modo de orientar a ação é indireto, visto que é mais adequada para que, a partir dela possamos direcionar nossos comportamentos ${ }^{(7)}$.

O comportamento moral (ético) ${ }^{(8)}$ varia com o tempo e o lugar, conforme as condições em que o homem organiza as práticas do trabalho, e quando as relações de produção são alteradas, pode haver modificações nas normas do comportamento coletivo no trabalho. 
Assim, a ética aplicada exige a aplicação da teoria ética normativa a problemas cotidianos que, para cada profissão, surge de seu propósito. Por conseguinte, os valores e as normas da profissão de enfermeiro constituem a base e o filtro a partir dos quais são tomadas as decisões éticas ${ }^{(9)}$. Estas requerem que sejam tomadas em benefício de pacientes ou da população sob a sua assistência, e de acordo com o Código de Ética dos Profissionais de Enfermagem.

O Código de Ética dos Profissionais de Enfermagem consiste em um conjunto de normas a serem respeitadas e cumpridas pelos profissionais e que norteiam a sua conduta. Enfatiza a qualidade da assistência de enfermagem como um direito do cidadão e um dever moral do profissional. Conforme preconiza em seu Artigo 22, do Capítulo IV ${ }^{(10)}$, as atividades desenvolvidas por seus profissionais devem ser exercidas com justiça, competência, responsabilidade e honestidade. Explicita também como prescrição moral, os princípios fundamentais da profissão, os direitos dos trabalhadores, suas responsabilidades, deveres e proibições.

Destarte, para que o enfermeiro desempenhe suas atividades gerenciais com respeito à dignidade $\mathrm{e}$ salvaguardando os direitos das pessoas, é imprescindível que sua tomada de decisão na função gerencial seja pautada não apenas nos valores éticos como "justiça, respeito às pessoas, honestidade, veracidade, sigilo e beneficência ${ }^{(11)}$, mas também nos valores da profissão. Conseqüentemente, a reflexão ética que envolve o trabalho gerencial do enfermeiro deve ser uma constante no cotidiano de sua prática profissional, uma vez que emergem dilemas e situações conflituosas que podem envolver diversos atores que dele fazem parte.

No que se refere às decisões éticas a serem tomadas, é importante ressaltar que "a gravidade da questão ética diz respeito à importância de que ela se reveste para quem vai tomar a decisão"(12). Assim,

Muitas são as questões éticas relacionadas ao gerenciamento de enfermagem, que, freqüentemente, assume a dualidade entre aquilo que pode ser feito e aquilo que deve ser realizado. Existem valores morais diversos; contudo, a prática da enfermagem depende dos recursos acessíveis para acessar as escolhas. Todavia, muitas vezes, há limites, e a deliberação diante do que fazer, do que é prioridade e de menor risco fica condicionada a processos predeterminados, oriundos das condições de trabalho ${ }^{(12: 22)}$.

Todavia, a fim de que o trabalho de enfermagem seja viabilizado de modo seguro, planejado, e de acordo com as necessidades do paciente, o enfermeiro gerencia o cuidado bem como exerce ações de cuidado direto. Seu processo de trabalho de gerência está orientado por uma finalidade imediata que é organizar o trabalho, e a mediata é prover condições para a realização do processo de cuidar individual e coletivo ${ }^{(13)}$.

Conseqüentemente, o uso dos instrumentos e funções gerenciais como planejamento, dimensionamento de pessoal, recrutamento e seleção, educação continuada, liderança, supervisão, e avaliação de desempenho possibilitam que o enfermeiro gerencie sua equipe adequadamente, e a conduza para a prestação de cuidados com a qualidade pretendida. Estes instrumentos viabilizam uma conduta ética no cuidado ao paciente, ou seja, uma assistência planejada, segura e livre de danos. Contudo, em decorrência de o enfermeiro assumir diversas funções gerenciais em instituições de saúde, tem havido polêmica na categoria, quanto à divergência entre o que se espera desse profissional sob o ponto de vista teórico e o que executa em seu cotidiano ${ }^{(14)}$. Essa divergência pode ocorrer por ser levado a tomar decisões fortemente influenciado pelo poder instituído, apesar de também ser influenciado pelo conhecimento e valores apreendidos durante a sua formação técnica, e também pelas questões que envolvem a sua sobrevivência no mercado de trabalho ${ }^{(15)}$.

Com relação ao contexto hospitalar, os profissionais de Enfermagem constituem sua maior força de trabalho ${ }^{(16)}$. No entanto, desempenham atividades freqüentemente marcadas por divisão fragmentada de tarefas; rígida estrutura hierárquica para o cumprimento de rotinas, normas e regulamentos; dimensionamento qualitativo e quantitativo de pessoal insuficiente - situações do exercício profissional que têm suscitado desmotivação de profissionais de enfermagem e elevado absenteísmo. Isso tudo nos mostra que o enfermeiro gerente do Serviço de Enfermagem depara-se com diversas dificuldades, dentre as quais salientam-se as relativas à estruturação dos serviços para o cuidado aos pacientes.

Há que se ressaltar que o trabalho desenvolvido pelos profissionais de Enfermagem é caracterizado como um processo contínuo e complexo. Portanto, é imprescindível que se tenham boas condições de trabalho que se referem a: ambiente adequado para a assistência e recursos físicos e materiais indispensáveis para o desenvolvimento de suas ações, além de recursos humanos suficientes, a fim de que a demanda seja atendida com qualidade, 
ou seja, com eficiência, eficácia e efetividade.

A escassez de recursos físicos e materiais tem sido um fator freqüentemente apontado como gerador de conflito para os profissionais de enfermagem nas instituições, pois existe uma demanda de cuidados aos pacientes que necessitam de atendimento, que nem sempre é realizado com a qualidade almejada, fato que suscita questões éticas. Neste sentido, a ética relacionada com a instituição diz respeito a como o trabalho é organizado no tocante aos recursos humanos e materiais, e, freqüentemente, em algumas instituições, os trabalhadores se vêem na contingência de terem que atuar com recursos materiais inadequados, de qualidade questionável e insuficientes em quantidade - situações que expõem os trabalhadores a riscos ocupacionais, ou os pacientes a riscos relativos à biossegurança ${ }^{(17)}$.

Tendo em vista a relação entre a ética e a organização do cuidado ${ }^{(17)}$, pode-se afirmar ainda que questões éticas que advêm de inadequadas condições de trabalho se refletem no cuidado prestado pelos profissionais ao paciente, mas também no relacionamento interpessoal entre profissionais de enfermagem, e entre estes e aqueles que são o objeto de seu cuidado.

Essas situações relatadas exigem que o enfermeiro mantenha um posicionamento consistente frente à instituição em que exerce a função de gerente do cuidado, ou seja, que tenha poder de argumentação, esteja embasado no código de ética dos profissionais de enfermagem e na legislação profissional vigente, e tenha ainda determinação, a fim de que possa viabilizar mudanças planejadas que venham a contribuir para a concretização de melhores condições de trabalho à categoria, e que redundem em adequada prestação de cuidados para indivíduos e à comunidade, de forma segura e de acordo com os padrões desejados de qualidade.

Entre as considerações que devem ser feitas na argumentação por melhores condições de trabalho, o enfermeiro pode enfatizar que quando se tratam de questões de déficit de pessoal para atender à demanda de cuidados aos pacientes, o princípio ético universal da beneficência é desrespeitado. Esse princípio refere-se às ações que devem ser implementadas na tentativa de se promover o bem ${ }^{(9)}$ dos pacientes. Porém, ainda que o profissional o deseje, demonstrando comportamento ético, nem sempre consegue contribuir para o alcance do bem estar dos pacientes, devido à impossibilidade de realizar-lhe os cuidados necessários, uma vez que requerem a sua presença junto ao paciente para se efetivarem. Esta não pode ser viabilizada devido ao fato dele estar responsável por uma quantidade excessiva de pacientes ao mesmo tempo, ou não ter condições de trabalho (recursos materiais, físicos e tecnológicos) para tal.

No que se refere às responsabilidades, o Código de Ética dos Profissionais de Enfermagem no seu Art. 16, Cap. III, diz que compete aos profissionais: "Assegurar ao cliente uma assistência de Enfermagem livre de danos decorrentes de imperícia, negligência ou imprudência" ". Assim, o enfermeiro tem poder de argumentação em relação ao fato de que a inexistência de condições adequadas de trabalho para profissionais de enfermagem pode incorrer em imprudência, algumas vezes involuntária, na prestação de cuidados, devido a fatores como a carga excessiva de trabalho e falta de organização e provimento de recursos no processo de cuidar. Além disso, as precárias condições laborais podem contribuir para insatisfação e falta de motivação de profissionais para o trabalho, desrespeito entre eles e superiores, gerando um comportamento individualista e não colaborativo.

Contudo, faz-se necessário questionar: se estes danos aos pacientes podem ser involuntários, mas decorrentes de falta de condições de trabalho, quem é responsável por eles? A resposta indubitável é que os gerentes de todos os níveis da instituição são co-responsáveis por esses danos aos pacientes, e também, por realizarem o planejamento global da instituição. Este deve prever condições de trabalho adequadas aos profissionais da saúde.

É importante enfatizar que a gerência de enfermagem pode transformar a realidade, porquanto tem poder de reorganizar o cuidado e o processo de trabalho. Contudo, as tecnologias gerenciais são importantes para a efetividade de processos e produtos do trabalho em saúde, mas requerem apoio políticoideológico para serem efetivas ${ }^{(18)}$. Por conseguinte, compete ao enfermeiro obter apoio de gestores quanto à viabilização de condições de trabalho, a fim de dirimir problemas e adequar recursos humanos e materiais para o cuidado aos pacientes, respeitando os princípios éticos universais referentes à dignidade humana, beneficência, não maleficência e justiça.

Neste sentido, não se pode esquecer que no setor de saúde gerenciam-se recursos materiais e humanos escassos, para necessidades ilimitadas ${ }^{(19)}$. Por conseguinte, o gerenciamento desses de maneira adequada, remete à responsabilidade pelo planejamento, a fim de que os objetivos operacionais sejam convergentes com a realidade sanitária das pessoas que utilizam esses serviços. Adicionalmente, deve-se 
considerar, ainda neste contexto, os componentes éticos do cuidar, "princípios éticos e os valores morais - hábitos, virtudes, atitudes, características e qualidades - que definem os comportamentos do cuidador"(20).

\section{CONSIDERAÇÕES}

O gerenciamento em Enfermagem é permeado por inúmeras variáveis: pessoais, da profissão, institucionais, econômicas, legais, políticas e éticas, que influenciam a prática do cuidado à saúde. Com o propósito de que este gerenciamento seja eficaz, é mister que o enfermeiro atue eticamente, porquanto a ética enquanto modo de ser expressa valores não apenas sociais, mas pessoais que são construídos, elaborados e introjetados ao longo da trajetória existencial, reflita, discuta e avalie com seus pares as possíveis soluções para os problemas, dilemas e situações conflituosas, de forma a cumprir com os objetivos da profissão e da instituição de saúde, e atender com qualidade às necessidades do paciente e da sua equipe.

Destarte, é imprescindível que a ação gerencial do enfermeiro e seu processo decisório seja fundamentada em princípios éticos. Assim, ao definir metas para sua prática gerencial, deve considerar os meios necessários para atingí-las, a fim de que possam ir ao encontro dos seus anseios, da filosofia de trabalho, da instituição onde atua, da essência da Enfermagem, e, principalmente, aos preceitos éticos universais referentes à preservação da dignidade humana, beneficência, não maleficência e justiça.

\section{REFERÊNCIAS}

1 Pires D. A estrutura objetiva do trabalho em saúde. In: Programa de Pós Graduação em Enfermagem/UFSC. Processo de trabalho em saúde: organização e subjetividade. Florianópolis: Papa-livros; 1999. p.25-48.

2 Luz MT. Práticas eficazes x modelos ideais: ação e pensamento na construção da integralidade. In: Pinheiro R, Mattos RA, organizadores. Construção da integralidade: cotidiano, saberes e práticas em saúde. Rio de Janeiro: UERJ/IMS; 2003. Pt1, p.7-34.

3 Lima MJ de. O que é enfermagem. $2^{\text {a }}$ ed. São Paulo: Brasiliense; 1994.

4 Brasil. Lei n. 7.498, de 25 de junho de 1986. Dispõe sobre a regulamentação do exercício da enfermagem e dá outras providências. Diário Oficial da República Federativa do Brasil, Brasília, 26 jun. 1986. Seção 1, p. 1.
5 Labronici, LM. Eros propiciando a compreensão da sexualidade das enfermeiras [tese]. Florianópolis (SC): Universidade Federal de Santa Catarina; 2002.

6 Cortes BA. Ética é limite. Trab Edu Saúde [periódico na Internet]. 2005 [acesso em 2006 Mai 27] (3): [19 páginas]. Disponível em: http:// www.revista.epsjv.fiocruz.br.

7 Cortina A, Navarro EM. Ética. São Paulo: Loyola; 2005.

8 Aranha MLA, Martins MHP. Filosofando: introdução à filosofia. $2^{\mathrm{a}}$ ed. São Paulo: Moderna; 1993.

9 Marquis BL, Huston CJ. Questões éticas e jurídicas. In: Administração e liderança em enfermagem: teoria e prática. $4^{\mathrm{a}}$ ed. Porto Alegre: Artmed; 2005: 399-418.

10 Conselho Federal de Enfermagem. Resolução COFEN $n^{\circ} 240 / 2000$. Aprova o Código de Ética dos Profissionais de Enfermagem [acesso em 2006 Mai 25]. Disponível em: http://www.portalcofen.gov.br/2007

11 Marcon PM, Polak YNS, Meier MJ. The Bioethics in the management of nursing work process: a reflexion. Online Braz J Nurs [periódico na Internet]. 2005 [acesso 2007 Feb 27]4(2). Disponível em: www.uff.br/nepae/ objn402marconetal.htm.

12 Massarolo MCKB, Fernandes MFP. Ética e gerenciamento em enfermagem. In: Kurcgant P, organizadores. Gerenciamento de enfermagem. Rio de Janeiro: Guanabara Koogan; 2005. p.14-25.

13 Felli VEA, Peduzzi M. O trabalho gerencial em enfermagem. In: Kurgant P, organizadores. Gerenciamento de enfermagem. Rio de Janeiro: Guanabara Koogan; 2005. p.1-13.

14 Trevizan MA, Mendes, IAC, Lourenço MR, Shinyashik, GT. Aspectos éticos na ação gerencial do enfermeiro. Rev Latino-am Enferm [periódico na Internet]. 2002 [acesso em 2007 Fev 27]10(1). Disponível em: http://www.scielo.br/ scielo.php.

15 Leopardi MT. A ética como fundamento decisório para a saúde como projeto humano. In: Programa de Pós Graduação em Enfermagem/UFSC. Processo de trabalho em saúde: organização e subjetividade. Florianópolis: Papa-livros;1999. p.153-8.

16 Barboza DB, Soler ZASG. Afastamentos do trabalho na enfermagem: ocorrências com trabalhadores de um hospital de ensino. Rev Lat-amEnferm. 2003;11(2):177-83

17 Lunardi VL, Lunardi Filho WD, Silveira RS, Soares NV, 
Lipinski JM. O cuidado de si como condição para o cuidado dos outros na prática de saúde. Rev Latino-am Enferm. 2004; 12(6):933-9.

18 Ermel RC, Fracolli LA. Processo de trabalho de gerência: uma revisão de literatura. Rev Esc Enferm USP. 2003;37(2):89-96.

19 Barrios SRL, Ferreira JHG, Tancredi, FB. Planejamento em saúde. Série Saúde e Cidadania [periódico na Internet].1998 [acesso em 2007 Fev 27]. Disponível em: http://www.saude.sc.gov.br/gestores/sala_de_leitura/ saude_e_cidadania/ed_02/index.html.

20 Restrepo MEM. La ética en el arte de cuidar. Investigación y Educación en Enfermería. Universidad de Antioquia [periódico na Internet] 2002 [acesso em 2007 Fev 27]. Disponivel em: http://tone.udea.edu.co/ revista/html/modules.php. 